\section{Unlocking ketones}

\section{By Michael J. Haas, Senior Writer}

Methylene groups, a ubiquitous structural feature of small molecule therapeutics, are notoriously nonreactive. This makes it challenging to modify them, a process vital to generating drug derivatives and new drug scaffolds.

Researchers at the University of Illinois at Urbana-Champaign (UIUC) think they have solved the problem. They have developed a chemical reaction that selectively oxidizes methylene groups and turns them into much more reactive ketones that can be converted to a wide range of other functional groups such as alcohols, ethers and esters. ${ }^{1}$ The technique could open new chemical space by allowing natural products and other structures to be oxidized at carbon sites that were previously difficult or impossible to modify with traditional medicinal chemistry techniques.

The technique already has attracted the attention of Pfizer Inc., which is exploring its use to identify metabolites of candidate drugs, according to the UIUC researchers.

Methylene groups are carbons bonded to two hydrogens and two other carbons, nitrogens and/or oxygens. In microorganisms and plants, biosynthetic enzymes readily oxidize methylenes on complex structures such as alkaloids. However, the enzymes are rarely used in the lab to oxidize methylenes because they act on a narrow range of structures and often give yields that are too small to be useful for subsequent chemical reactions.

Ketones and other oxidized groups can be incorporated into specific sites in a compound via total synthesis-the process of building a complex compound from simpler molecules that contain the desired functional groups. But total synthesis is a lengthy and laborious approach that is not well suited to expanding chemical space or producing multiple analogs of a core scaffold.

Thus, there has been a long-standing need for a broadly useful medicinal chemistry technique that could oxidize methylenes without relying on biosynthetic enzymes or total synthesis.

In two reports in the past three years, the UIUC researchers showed that an iron-based catalyst could oxidize methine-a carbon bonded to one hydrogen and three other carbons, nitrogens and/or oxygens-on a wide range of structures., ${ }^{2,3}$

Because methylene groups tend to be more sterically accessible than methines and are prevalent in the ring structures found in drug-like molecules, the UIUC team set out to determine whether their ironcatalyzed reaction could also oxidize methylene carbons.

The method readily oxidized methylenes on small molecules such as substituted cyclohexanes. In addition, the locations of the oxidation reactions were predictable. This allowed the team to develop a set of selectivity rules for determining where oxidations were most likely to occur on more complex structures.

Next, they used the method to oxidize the bactericidal terpenoid pleuromutilin and other biologically active terpenoids. Indeed, the major products were those predicted by the team's selectivity rules.

Moreover, the researchers found that the sites oxidized by their reaction were different than those oxidized by biosynthetic enzymes (see Figure 1, "The path to new chemical structures").

"Reactivity that is complementary to enzymatic systems offers the potential for investigating previously unexplored chemical space that would otherwise be synthetically challenging to access," said team leader M. Christina White, associate professor of chemistry at UIUC.

White conducted the study with Mark Chen, a former graduate student in her lab. Data were reported in Science.

"The installation of a ketone is a particularly useful functional group, as chemists view such groups as a starting point for countless synthetic transformations to yield more elaborate molecules," said Mark Plummer, associate research fellow in antibacterial medicinal chemistry at Pfizer.

\section{Singing in key(tone)}

The UIUC team's method could help streamline drug discovery research on natural products by allowing researchers to modify carbon sites that had been inaccessible to many medicinal chemistry techniques.

"This is a very fine study and the work is groundbreaking," said Andrew Myers, chair of chemistry and chemical biology at Harvard University. "I imagine its greatest utility might well be the functionalization of valuable, complex molecules such as bioactive natural products to enable new analogs to be prepared and evaluated."

According to Andrew Combs, executive director of chemistry at Incyte Corp., the chemical reaction could be used to make libraries of natural product analogs, synthesize entirely new chemotypes or optimize lead compounds. "You could take some intermediate scaffold you've synthesized in a few steps, then put functionalities on it" instead of having to build in those functional groups from scratch, he said.

Plummer agreed. Natural product analogs synthesized with the UIUC technique "would possess new and different biological activity, thereby furthering the potential of natural product-based medicine discovery," he said.

"I suspect this work will be rapidly implemented by many laboratories-including my own-that are working on routes to complex bioactive natural products," said Gregory Roth, associate professor and director of medicinal chemistry and exploratory pharmacology at the Sanford-Burnham Institute for Medical Research.

He added that catalytic transformations are also important for drug manufacturing. "I think many chemical development laboratories will incorporate the knowledge generated by the White research group into their efforts," he said. 
In addition to expanding the chemical space, the UIUC team's reaction method could speed the exploration of that space compared with the total synthesis method, said Paul Wender, professor of chemistry at Stanford University.

Without the new chemical reaction, he noted, a chemist would have to introduce the oxidized site early in the total synthesis process. Then it would be necessary to attach a structure to that site-a protecting group-to prevent it from reacting in subsequent steps of the synthesis. And then the protecting group would have to be removed later.

The problem is that protecting groups often limit the chemistry that can be done elsewhere on the molecule, and adding and removing such groups tacks on additional steps to a reaction sequence.

"The UIUC team's method could contribute to high-speed synthesis" by reducing the number and amount of reagents, and the time and effort required to synthesize a desired structure, Wender told SciBX. "Most people who are investigating therapeutic molecules would appreciate having the ability to make analogs or diversify a lead compound" at the end stage of the drug discovery process, when the activity of a core structure or scaffold has been established.

Combs said there is room to improve the chemical reaction. The method's ability to selectively oxidize a desired methylene site was only moderate in some of the example reactions presented in the Science paper, he said.
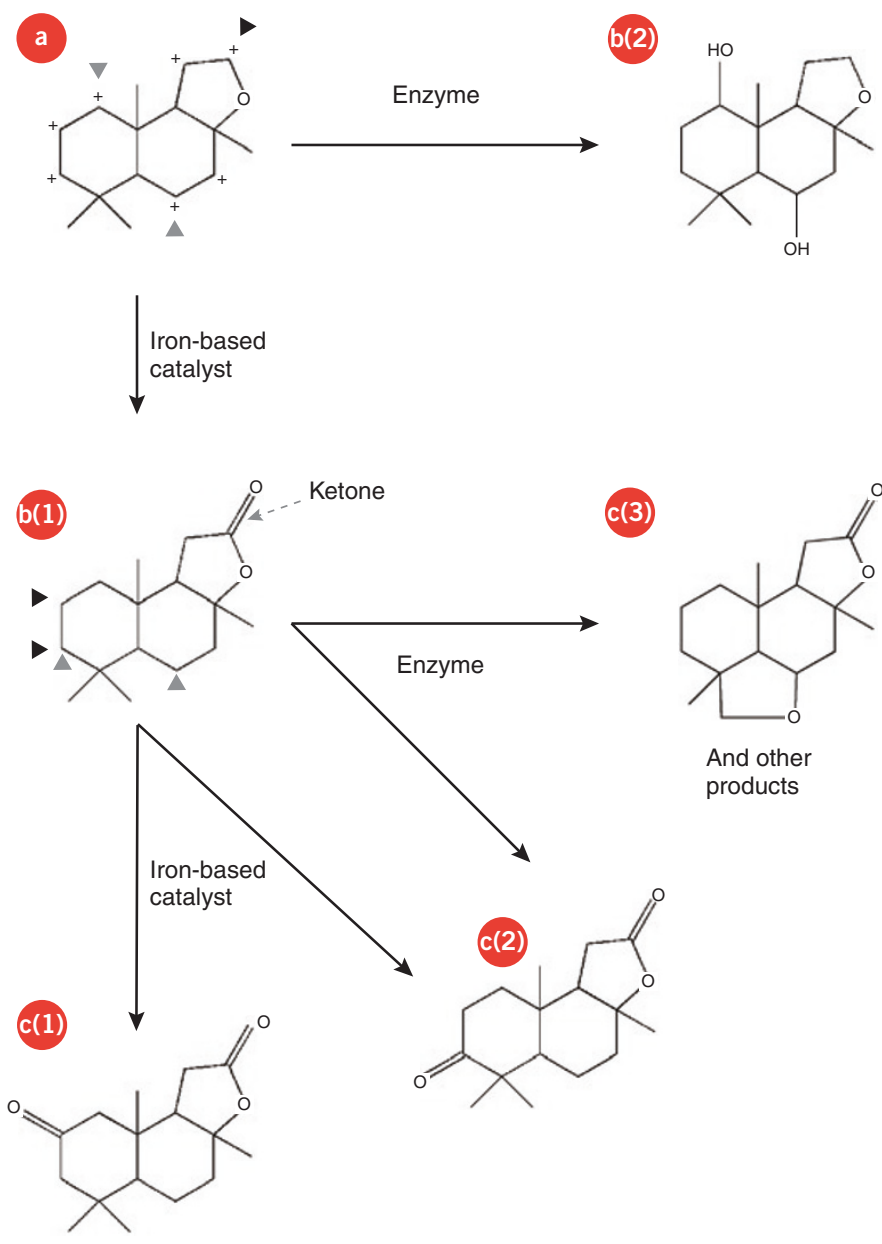

The result, Combs said, is that "there isn't just one major product from a reaction. There are several other oxidation products, which the authors label as 'minor', ranging from $15 \%-37 \%$ of overall yields." $\mathrm{He}$ did not consider those yields as minor but noted that the secondary products might be useful to chemists interested in diversifying a scaffold or exploring new chemotypes.

Wender was more sanguine about the method's selectivity. "In some cases it's acceptable; in others, it's spectacular," he said. "The team provides an inspirational set of examples of the reaction's selectivity and a primer on how to apply it to many situations. This is important because you can infer from what they did and apply their selectivity rules to a compound you might have in your own lab."

Combs also noted that the UIUC team didn't use the method to modify any nitrogen-containing molecules. "The last time I saw a drug without a nitrogen was a long time ago," he said. Thus, Combs wanted to see example reactions on molecules containing different classes of nitrogen functionalities to determine which of those functionalities might require protection from oxidation.

Plummer agreed that it would be important to assess the method's effect on non-methylene functional groups because it was such a powerful oxidation system. "Oxidizable functionalities such as primary and secondary alcohols and basic amines would have to be protected so as not to be targets of the oxidation," he said.

White agreed, noting that her team successfully used protecting groups to prevent the oxidation of secondary amines in their previous study of methine oxidation ${ }^{2}$ and has found that some types of amides survive without protecting groups.

"We are currently working on a catalyst that would allow us to tolerate" a range of $\mathrm{N}$-containing functional groups without having to protect them, she said.

\section{O to go}

White said her team is now working to optimize the reaction conditions.

Planned studies include the total synthesis of the cancer drug paclitaxel, starting with a hydrocarbon skeleton and using the reaction

Figure 1. The path to new chemical structures. A chemical reaction developed by University of Illinois at Urbana-Champaign researchers uses an iron-based catalyst to oxidize methylenes at sites complementary to those oxidized by biosynthetic enzymes. The reaction turns methylene groups into ketones, a useful starting point for multiple synthetic transformations.

In a paper in Science, the researchers described using the ironbased catalyst on ambroxide [a], which led to methylene oxidation primarily at one $(\boldsymbol{\Delta})$ of seven possible sites (+) to yield sclareolide $[\mathbf{b}(\mathbf{1})]$ as the main product. In comparison, a biosynthetic enzyme oxidized ambroxide at two other sites simultaneously $(\Lambda)$ to yield 1,6-dihydroxy-sclareolide $[\mathbf{b}(2)]$ as the main product.

Using the iron-based catalyst on sclareolide led to oxidation primarily at either of two sites $(\mathbf{A})[\mathbf{b}(\mathbf{1})]$ to yield 2-oxo-sclareolide [c(1)] and 3-oxo-sclareolide [c(2)] as main products. By contrast, a biosynthetic enzyme oxidized sclareolide at one of two or more possible sites (A) $[\mathbf{b}(\mathbf{1})]$ to yield 3-oxo-sclareolide [c(2)], $\mathrm{O}^{6}$-sclareolide [c(3)] and no less than four other products 


\section{ANALYSIS}

method to introduce the oxidation.

She added that her team's iron-based catalyst is now commercially available from Strem Chemicals Inc. as the White-Chen catalyst.

Pfizer would not comment on the results of its studies with the UIUC chemical reaction. But according to White, the pharma is exploring the use of the method to identify metabolites of drug candidates found in standard oxidative enzyme assays. That work is being conducted without a licensing agreement between UIUC and Pfizer, she said.

The findings reported in Science are patented by UIUC. The university is open to licensing and partnering opportunities, White said.

Haas, M.J. SciBX 3(6); doi:10.1038/scibx.2010.171

Published online Feb. 11, 2010

\section{REFERENCES}

1. Chen, M. \& White, M.C. Science; published online Jan. 28, 2010; doi:10.1126/science.1183602

Contact: M. Christina White, University of Illinois at UrbanaChampaign, Urbana, III. e-mail: white@scs.uiuc.edu

2. Chen, M. \& White, M.C. Science 318, 783-787 (2007)

3. Vermeulen, N. et al. Tetrahedron 65, 3078-3084 (2009)

\section{COMPANIES AND INSTITUTIONS MENTIONED}

Harvard University, Cambridge, Mass.

Incyte Corp. (NASDAQ:INCY), Wilmington, Del.

Pfizer Inc. (NYSE:PFE), New York, N.Y.

Sanford-Burnham Institute for Medical Research, La Jolla, Calif. Stanford University, Stanford, Calif.

Strem Chemicals Inc., Newburyport, Mass.

University of Illinois at Urbana-Champaign, Urbana, III. 